\title{
Evaluación del riesgo de negocio de las empresas que cotizan en el índice sustentable de la Bolsa Mexicana de Valores
}

\section{(Business risk assessment of the companies on the Mexican Stock Exchange's sustainable index)}

\author{
José Antonio Morales Castro ${ }^{1}$
}

\begin{abstract}
Resumen:
En este trabajo se evaluó el cambio de riesgo de negocio de 20 empresas que integran el índice sustentable de la Bolsa Mexicana de Valores (BMV), a través del coeficiente de beta desapalancada. Para ello se compararon el periodo previo versus el periodo posterior a la incorporación de las compañías al índice sustentable de la BMV. Se utilizó información financiera, precios de cierre de las acciones y del índice bursátil por un periodo de 234 semanas de cada una de las 20 empresas; posteriormente se calculó la diferencia estadística entre las medias de coeficiente beta desapalancada entre los dos periodos, y se realizó una prueba de hipótesis para evaluar el cambio de riesgo. Se encontró que de las 20 empresas, 12 disminuyeron en su coeficiente de beta desapalancada, los hallazgos sugieren que no es suficiente que las empresas se certifiquen como sustentables para reducir el riesgo de negocio.
\end{abstract}

Palabras clave: riesgo de negocio, empresas sustentables, Bolsa Mexicana de Valores

\begin{abstract}
:
This work evaluated the business risk change of 20 companies included on the sustainable index of the Mexican Stock Exchange (BMV). The unlevered beta coefficient was used for this analysis. Two periods were compared: the one before, and the one after including the companies on the index. For each of the 20 companies it was used financial information, stocks closing prices and the stock market index value, over a period of 234 weeks. Then, considering the two periods, the statistical difference between the unlevered beta coefficient averages was calculated. Finally, a hypothesis proof was made to evaluate the business risk change. It was found that for 12 out of the 20 companies, the unlevered beta coefficient suffered a reduction. The findings suggest that it is not enough for the companies to certify as sustainable in order to reduce its business risk.
\end{abstract}

Keywords: Business risk, sustainable companies, Mexican Stock Exchange.

\section{Introducción}

El problema de la mala calidad ambiental se consideraba exclusivo de los países desarrollados industrialmente, sin embargo en la actualidad ya alcanzó a todo el mundo, la erosión de los suelos, la baja calidad del agua, la deforestación y como consecuencia, ha aumentado los riesgos en el resguardo de la vida y entorno humano, los gobiernos para mitigar los efectos crean leyes que restringen la emisión de contaminantes y por consecuencia una probable declinación en la productividad y márgenes de rentabilidad empresarial por las restricciones impuestas (Ponce y Pérez, 2007; Páez, 2013 y Valderrábano et. al., 2011).

\footnotetext{
${ }^{1}$ Instituto Politécnico Nacional, Escuela Superior de Comercio y Administración unidad Tepepan, Ciudad de México, México (jmorales@ipn.mx).
} 
Para dar solución a este problema de contaminación en el ámbito financiero surgen los índices bursátiles sustentables que incorporan empresas que deben certificarse con tres áreas fundamentales, (1) cuidado de manejo y uso de recursos naturales, (2) responsabilidad social y (3) el gobierno corporativo, en el manejo de los recursos naturales se incluyen los impactos en el medio ambiente. A estas empresas también se les conocen como Responsabilidad Social Corporativa por sus siglas RSC.

En diciembre de 2011 la Bolsa Mexicana de Valores inició la operación del Índice de Precios y Cotizaciones (IPC) sustentable, el cual incluye las empresas que estando listadas en esta bolsa de valores, destacan en materia de sustentabilidad de acuerdo a los principios definidos en el Pacto Global de la ONU, donde se evalúan tres áreas: (1) manejo y uso de recursos naturales, (2) responsabilidad social con sus principales grupos de interés (empleados, proveedores, clientes y comunidad en que se desempeñan y (3) gobierno corporativo, estas empresas deben ubicarse en o por encima del promedio nacional de calificación conjunta de medio ambiente, responsabilidad social y gobierno corporativo de las empresas nacionales evaluadas por los calificadores independientes.

La administración contribuye para que las empresas sean sustentables en la medida que los administradores al tomar decisiones en áreas de financiamiento e inversión en las empresas, históricamente han contemplado varios de los aspectos que comprenden las áreas de la sustentabilidad, por ejemplo en el segmento de responsabilidad social, en 1899 con Andrew Carnegie fundador del conglomerado U.S. Stell publicó un libro llamado el Evangelio de la Riqueza donde se postulaba que los miembros más afortunados de la sociedad debían ayudar a los menos afortunados (Van Horne, 1997; Gitman, 2007; Stoner, Freeman y Gilbert, 1996 y Koontz, Weihrich y Cannice, 2012). Durante la década de 1990 se intensificaron los esfuerzos por el cuidado el medio ambiente estableciéndose comisiones y organizaciones especiales en pro del cuidado del ambiente, como son las de la ONU, OMC, CEPAL, por mencionar algunas (Valderrábano, 2011; Stoner, Freeman y Gilbert, 1996 y Páez, 2013).

\subsection{Marco teórico empírico}

El objetivo de las empresas es crear valor financiero para los accionistas a través del incremento del precio de la acción y el pago de dividendos, en el largo plazo se busca que las corporaciones perduren a lo largo del tiempo, para lograr recuperar la inversión que han hecho en diversos activos, es lo que distingue a las empresas sostenibles o sustentables, (Organización Internacional del Trabajo, 2007, p. vi). Para que las compañías adopten el mote de sustentables cumplen con el modelo al que se le ha denominado Responsabilidad Social Corporativa (RSC), a través del cual fortalecen su cadena de valor y su relación con los colaboradores y asociados a la 
empresa, como son los grupos de empleados, clientes, proveedores, acreedores, autoridades fiscales y gubernamentales, propietarios y cualquier otro que tenga una relación directa con la empresa, con el objetivo de asegurar a las futuras generaciones una perdurabilidad adecuada. (Espino, 2009 y Almagro, 2009), de esa manera las empresas logran mantenerse funcionando en su medio ambiente y generando valor para sus accionistas.

Las empresas sustentables crean una reputación positiva en todas las partes que se relacionan con ella, lo que se debería traducirse en flujos de efectivo estables y crecientes, impactando en la percepción que tienen los inversores sobre el precio de la acción en los mercados bursátiles. Aunque al respecto no hay consenso en las investigaciones empíricas Margolis y Walsh (2001), Orlitzky, Schmidt y Rynes (2003) analizan la relación entre el comportamiento social de la empresa y los resultados financieros, señalan que después de 30 años y 95 estudios empíricos, la relación entre comportamiento social de las empresas y los resultados financieros sigue siendo confusa.

Cuando las empresas se han certificado como sustentables impactan en su nivel de riesgo y a su vez en el precio de sus títulos accionarios, aunque la percepción que tienen los inversionistas para determinar el precio de una acción en los mercados financieros, no basta esta característica, por ello escudriñan los estados financieros de la compañía, identifican las tendencias de la industria, pronostican las utilidades futuras y los dividendos que percibirán, (Berk y Demarzo, 2008; Weston y Brigham, 1993; Brealey y Myers, 1998 y Nieto y Fernández, 2004). El análisis para determinar los flujos de efectivo incluye tres áreas, (1) el análisis económico que supone la evaluación del estado general de la economía (política fiscal, política monetaria, inflación, gastos de consumo, inversiones empresariales, comercio exterior y tipos de cambio) y de sus efectos potenciales sobre los resultados empresariales, (2) el análisis industrial que se enfoca al ramo en que opera la compañía en estudio, su posición respecto a los principales competidores del sector, y (3) el análisis fundamental que profundiza en la situación financiera y los resultados de operación de una compañía específica así como en el comportamiento esencial de sus acciones comunes, (Gitman y Joehnk, 1997; Little y Rhodes, 1992 y Malkiel, 1995).

\subsubsection{La administración de riesgos en las empresas sustentables}

Una de las características de las empresas de los índices bursátiles sustentables es que usan más eficientemente los recursos (o disminuyendo los niveles de consumo de los materiales que emplean) y reduciendo la contaminación asociada a sus operaciones. "Adoptar modelos de consumo sostenible en toda la cadena valor redunda en una mayor resistencia a los choques con el ambiente exterior, dado que mitiga el impacto de los riesgos ante una posible escasez y/o incremento de los precios en ciertos recursos" (Senior et. al., 2007: 485). 
En ese mismo sentido coinciden (Lacruz, 2005; Murillo, Garcés y Rivera, 2004; Centro Empresarial de Inversión Social, 2004; Deloitte, 2011; Senior, Narváez, Fernández y Revilla, 2007), quienes explican que existen estudios que comprueban que estas empresas obtienen tasas de retorno a sus inversiones muy superiores a las expectativas como resultado de la reducción de costos de producción a través del descenso de gastos e improductividad. Aunque las flujos de efectivo dependen también del sector al que pertenece la empresa, la existencia de mercados monopólicos, la productividad e innovación que imponen las organizaciones con que se relacionan las empresas como son los establecimientos académicos, asociaciones comerciales y organizaciones relacionadas con la cadena productiva, cambios de ejecutivos y oscilaciones del ciclo de negocio (Porter y Kramer, 2006 y Porter y Kramer, 2011). Las empresas sustentables se enfocan en su cadena valor o ciclo de negocio, lo que significa que la empresa se preocupa desde sus proveedores de materias primas hasta los consumidores finales del producto. Lo cual se traduce en el diseño de sistemas de monitoreo del cumplimiento de estándares (ambientales y sociales) por parte de los proveedores. En la medida en que la información relevante de las empresas es transparente se disminuyen sustancialmente los riesgos de situaciones de fraudes en las empresas y su multiplicación en la economía global (Nuñez, 2003), por ello se puede considerar que las empresa que se certifican como sustentables debieran tener menores nivele de riesgo de negocio.

\subsubsection{Estudios empíricos sobre el riesgo empresarial}

Sharpe (1964) desarrolló el modelo de valuación de activos financieros (CAPM, por sus siglas en ingles) el cual estima el rendimiento mínimo esperado o exigible de un activo en función de su riesgo de mercado o sistemático. Posteriormente Lintner (1965) y Mossin (1966) desarrollan trabajos con modelos donde estiman el rendimiento mínimo esperado o exigible en función del riesgo sistemático o de mercado el cual es medido por el coeficiente beta. Hamada (1969) estudia cómo se determina el coeficiente beta, y descompone el modelo de cálculo y encuentra la beta desapalancada, posteriormente Damodaran (1998), Velez (2011), explican que existen dos factores sobre los cuales la acción de la empresa basa su sensibilidad (coeficiente beta) con respecto al mercado, uno es el riesgo de negocio u operativo el cual representa el riesgo que tiene la empresa por su naturaleza-apalancamiento operativo, volatilidad de la demanda, etcétera, por otro lado se encuentra el factor riesgo financiero, el cual representa la situación financiera de la empresa derivado de su política de financiamiento, determinado a través del nivel de apalancamiento empleado por la corporación.

El riesgo de negocio está relacionado con la variabilidad de los ingresos, el cual depende del riesgo de ventas y el riesgo operativo, el riesgo de ventas se encuentra afectado por la elasticidad de la demanda respecto al producto, el comportamiento cíclico de los ingresos y la estructura competitiva de la industria. "En lo que se refiere al riesgo operativo depende de la estructura de 
costos fijos y variables, cuanto mayor sean los costos fijos en relación a los costos variables, mayor es la incertidumbre en relación a las utilidades y flujos de efectivo" (Con base en Vargas, 2011, p. 125), por ello la beta desapalancada mide específicamente el riesgo que proviene de la empresa.

El valor del coeficiente beta desapalancada está en relación a los índices bursátiles empleados para determinar el rendimiento de mercado, (López, 2000), a las variables macroeconómicas que influyen en los mercados financieros (Vargas, 2011; Vélez, 2011; Hernández y Mora, 2010; Moscoso et. al., 2012). Forética (2011), estudió 19 compañías del índice Dow Jones sustentable, y encontró el coeficiente de las betas desapalancadas presentan un riesgo ligeramente menor que el mercado, el 58\% de las compañías tiene una beta desapalancada inferior a su industria.

En lo que se refiere al comportamiento del coeficiente de las betas desapalancadas de los diferentes sectores de empresas en los países emergentes, se encontraron investigaciones desarrolladas por Hernández y Mora (2009) y Vargas (2011).

Hernández y Mora (2009) realizan una serie de ajustes en el cálculo de las betas para el entorno en que desarrollan operaciones las empresas de Costa Rica y presentan los valores de las betas desapalancadas para diez sectores, las cuales se observan en la Tabla 1.

Tabla 1. Coeficiente de las betas desapalancadas para las empresas en Costa Rica.

\begin{tabular}{|c|c|}
\hline \multicolumn{2}{|c|}{ Costa Rica } \\
\hline Sector & Beta desapalancada \\
\hline Bebidas & 2.64 \\
\hline Biotecnología & 4.33 \\
\hline TV por cable & 2.79 \\
\hline Software/Computadoras & 4.31 \\
\hline Comercio electrónico & 4.85 \\
\hline Servicios educativos & 3.05 \\
\hline Alimentos procesados & 1.84 \\
\hline Confitería & 1.66 \\
\hline Servicios médicos & 2.89 \\
\hline Periódicos & 1.56 \\
\hline
\end{tabular}

Fuente: Hernández R. M. y Mora E. R. (2009), p. 83.

En la Tabla 2 se presenta el valor del coeficiente beta desapalancada de cinco sectores de Pymes de Bolivia, es de resaltar que sus valores son más altos que las betas desapalancadas de las empresas que cotizan en bolsa de valores (véase Tabla 3) lo que hace pensar que las pyme tienen mayores niveles de riesgo de negocio que las empresas que cotizan en bolsa de valores. 
Tabla 2. Valores de coeficiente de beta desapalancada de Bolivia

\begin{tabular}{|l|c|}
\hline Betas para Pymes de Bolivia & $\begin{array}{c}\text { Beta } \\
\text { desapalancada }\end{array}$ \\
\hline Textiles, prendas de vestir, cueros & 1.3233 \\
Metálmecanica, materiales y equipos & 1.4448 \\
Aserraderos y productos de madera & 1.4935 \\
Alimentos, bebidas y tabaco & 0.8599 \\
Productos químicos, Lab. Farmaceúticos y plasticos & 1.3896 \\
\hline
\end{tabular}

Fuente: Vargas S. A. (2011), p. 131.

En la Tabla 3 se presentan los coeficientes de las betas desapalancadas de las empresas de varios países de economías emergentes de América Latina, entre ellos Brasil, Chile, México, Colombia y también de EUA, donde se usaron datos de 1000 días de cotización de precios de las acciones y de los índices bursátiles correspondientes de cada país los cuales se obtuvieron de la base de datos de Reuters con cierres a diciembre de 2014 y se usó la clasificación sectorial de GICS (Global Industry Classification Standard).

Tabla 3. Coeficientes de beta desapalancada de países Latinoamericanos y EUA

\begin{tabular}{|l|c|c|c|c|c|c|}
\hline \multicolumn{1}{|c|}{ Sector } & BRASIL & CHILE & MÉXICO & $\begin{array}{c}\text { COLOMBIA } \\
\text { (IGBC) }\end{array}$ & PERU & EUA \\
\hline Bancos & & & & \\
Servicios financieros diversificados & 0.21 & 0.16 & 0.25 & 0.09 & 0.17 & 0.23 \\
Energía & 0.32 & 0.06 & 0.88 & 0.38 & & 0.65 \\
Comida y ventas al por menor productos basicos & 0.26 & 0.65 & 0.72 & 0.54 & 0.60 & 0.57 \\
Comida, bebidas y tabaco & 0.39 & 0.38 & 0.47 & 0.51 & & 0.55 \\
Materiales & 0.45 & 1.22 & 0.83 & 0.41 & 0.77 & 0.84 \\
Transportación & 0.37 & 0.80 & 0.55 & 0.54 & & 0.85 \\
Empresas servicios publicos & 0.27 & 0.59 & 0.52 & 0.36 & 0.63 & 0.32 \\
Seguros & 0.55 & & & & & 0.31 \\
Bienes raíces & 0.35 & 0.53 & & & & 0.73 \\
Software y servicios & 0.36 & 0.72 & & & & 0.88 \\
Bienes de consumo duradero y de vestir & 0.36 & 0.62 & & & & 0.96 \\
Bienes de capital & 0.33 & 0.61 & 0.57 & 0.32 & 0.54 & 0.92 \\
Servicios al consumidor & 0.45 & & 0.66 & & & 0.84 \\
Ventas al por menor & 0.64 & 0.44 & 0.46 & & & 0.75 \\
Productos del hogar y personales & 0.51 & & 0.61 & & & 0.61 \\
Servicios de telecomunicaciones & 0.28 & 0.37 & 0.51 & 0.28 & & 0.33 \\
Equipos de salud y servicios & 1.07 & 0.44 & & & & 0.70 \\
Farmacia y biotecnología & & 0.52 & 0.75 & & & \\
\hline
\end{tabular}

Fuente: Buenaventura, (2014), Departamento de Estudios Contables y Financieros, Facultad de Ciencias Administrativas y Económicas de La Universidad ICESI de Cali Colombia.

En la Tabla 3 se observa que cada sector tiene diferente riesgo operativo (beta desapalancada) y varía según el país, por ejemplo en el caso del sector de bienes de capital las empresas de EUA tienen mayor riesgo que las compañías de los países latinoamericanos, en contraste en el caso 
del sector de telecomunicaciones en México tiene mayor nivel de beta desapalancada que los demás países.

Por ello se considera que en el nivel de riesgo operativo influyen varios factores como son el tamaño de la empresa, la fase del ciclo económico, las perspectivas de dominio de las compañías en el sector y las variables macroeconómicas (López, 2000; Vargas, 2011; Vélez, 2011; Hernández y Mora, 2010; Moscoso et. al., 2012), por ejemplo en el caso del riesgo operativo de los bancos en EUA es similar al de los otros países tal como se muestra en la tabla 3 que corresponde al 2014, pero si se calculara la beta desapalancada para el año 2009, seguramente los bancos de EUA tendrían niveles de riesgo operativo más elevados por los efectos de la crisis de 2008 que impacto negativamente los resultados de las instituciones financieras de EUA.

\subsection{Descripción del problema}

Aquellas empresas que actúan de manera responsable, es decir, que escuchan a sus stakeholders y actúan tomando en cuenta sus expectativas, que respetan el medio ambiente donde interactúan, que desarrollan relaciones de largo plazo con sus proveedores, que satisfacen las necesidades de sus clientes sin poner en riesgo la calidad de los productos o servicios que mantienen a sus colaboradores motivados, etc., tienen menores riesgos de insustentabilidad y, por lo tanto, son vistas como empresas más seguras (Perera, 2011: 163), es decir que su riesgo de negocio es menor.

Existen diversas investigaciones sobre el comportamiento del riesgo, medido a través del coeficiente beta, de las empresas que forman parte del índice sustentable de las bolsas de valores, según Vargas (2011) el coeficiente beta de las empresas de los países emergentes es diferente al de los países desarrollados, por otra parte Forética (2011), encontró en una muestra de 19 empresas del índice sustentables del Dow Jones de EUA que el riesgo especifico (beta desapalancada) de las empresas es ligeramente inferior que el mercado. Entre los factores que determinan el comportamiento de las empresas en la bolsa se encuentran los resultados financieros según (Berk y Demarzo, 2008; Weston y Brigham, 1993; Brealey y Myers, 1998 Gitman y Joehnk, 1997; Little y Rhodes, 1992 y Malkiel, 1995).

Los resultados del comportamiento de las empresas sustentables en la bolsa de valores no han sido concluyentes, según Margolis y Walsh (2001), Orlitzky, Schmidt y Rynes (2003) la relación entre el comportamiento social de las empresas y los resultados financieros siguen siendo confusos, por otra parte Porter y Kramer (2006) y Porter y Kramer (2011), explican que los resultados financieros de las empresas no depende únicamente de cumplir con la certificación de empresas sustentables, existen otros factores que determinan el riesgo de las empresas, entre 
ellos se encuentran los cambios de ejecutivos de las empresas, las oscilaciones del ciclo de negocio que afectan la productividad de la empresa.

En el caso de las empresas que se incorporaron en el índice sustentable de la Bolsa Mexicana de Valores, algunas pertenecen al sector de la construcción y dado que durante el año de 2014 los precios de sus acciones han variado afectando su valor de coeficiente beta, por ello se plantean las siguientes preguntas de investigación:

\section{Principal}

¿Existe diferencia estadística en los valores medios del coeficiente de beta desapalancada que mide el riesgo de negocio en las empresas que cotizan en la BMV antes versus después de incorporarse al IPC sustentable?

\section{Hipótesis}

Para evaluar la diferencia del nivel de coeficiente beta entre los dos periodos se realizó una prueba de hipótesis para contrastación de las medias, donde $\mathrm{H}_{0}$ y $\mathrm{H}_{1}$ es la hipótesis nula y la hipótesis alternativa respectivamente:

\section{Principal}

$\mathbf{H}_{0}$. No existe diferencia estadística en los valores medios del coeficiente de beta desapalancada que mide el riesgo de negocio en las empresas que cotizan en la BMV antes versus después de incorporarse al IPC sustentable.

$\mathbf{H}_{1}$. Existe diferencia estadística en los valores medios del coeficiente de beta desapalancada que mide el riesgo de negocio en las empresas que cotizan en la BMV antes versus después de incorporarse al IPC sustentable.

\section{Metodología}

\subsection{Métodos y materiales}

En esta investigación, las empresas seleccionadas y que integran IPC sustentable de la Bolsa Mexicana de Valores (BMV) que inició en el año 2011, se muestra en el tabla 4, se excluyeron los bancos, dado que su análisis financiero es diferente a las demás empresas.

\subsubsection{Materiales}

Para el cálculo de la beta, se utilizaron los precios de cierre de las acciones de las empresas que integran el IPC sustentable de la BMV y del IPC, con lo cual se determinaron los rendimientos y las betas semanales considerando un periodo de tiempo desde 2009 a 2014, equivalente 234 
semanas, también se obtuvieron los estados financieros de la base de datos de Economatica para determinar el nivel de apalancamiento financiero, y con ello calcular la beta desapalancada.

Tabla 4. Empresas del IPC sustentable de la Bolsa Mexicana de Valores

\begin{tabular}{|c|c|c|}
\hline Núm. & $\begin{array}{ll}\text { Clave } & \text { de } \\
\text { cotización }\end{array}$ & Razón social \\
\hline 1. & $A C^{*}$ & ARCA CONTINENTAL, S.A.B. DE C.V. \\
\hline 2. & ALFAA & ALFA, S.A. DE C.V. \\
\hline 3. & AMXL & AMÉRICA MÓVIL, S.A.B DE C.V. \\
\hline 4. & ASURB & GRUPO AEROPORTUARIO DEL SURESTE, S.A.B. DE C.V. \\
\hline 5. & BIMBOA & GRUPO BIMBO, S.A.B DE C.V. \\
\hline 6. & CEMEXCPO & CEMEX, S.A.B DE C.V. \\
\hline 7. & COMERCIUBC & CONTROLADORA COMERCIAL MEXICANA, S.A.B. DE C.V. \\
\hline 8. & FEMSAUBD & FOMENTO ECONÓMICO MEXICANO, S.A.B. DE C.V. \\
\hline 9. & GEOB & CORPORACIÓN GEO, S.A.B. DE C.V. \\
\hline 10. & GMEXICOB & GRUPO MÉXICO, S.A.B. DE C.V. \\
\hline 11. & GMODELOC & GRUPO MODELO, S. A. DE C.V. \\
\hline 12. & $\mathrm{HOMEX}^{*}$ & DESARROLLADORA HOMEX, S.A.B. DE C.V. \\
\hline 13. & $\mathrm{ICA}^{*}$ & EMPRESAS ICA, S.A.B. DE C.V. \\
\hline 14. & KIMBERA & KIMBERLY-CLARK DE MÉXICO, S.A.B. DE C.V. \\
\hline 15. & KOFL & COCA-COLA FEMSA, S.A.B DE C.V. \\
\hline 16. & MEXCHEM $^{*}$ & MEXICHEM, S.A.B. DE C.V. \\
\hline 17. & OMAB & GRUPO AEROPORTUARIO DEL CENTRO NORTE, S.A.B. DE C.V. \\
\hline 18. & PE\&OLES* & INDUSTRIAS PEÑOLES, S.A.B DE C.V. \\
\hline 19. & $\mathrm{URBI}^{*}$ & URBI DESARROLLOS URBANOS, S.A.B. DE C.V. \\
\hline 20. & WALMEX V & WAL-MART DE MÉXICO, S.A.B. DE C.V. \\
\hline
\end{tabular}

Fuente: Elaboración propia con datos de la Bolsa Mexicana de Valores

Posteriormente se separaron en dos periodos de tiempo, el primero de octubre de 2009 hasta diciembre de 2011 y el segundo de enero de 2012 hasta marzo de 2014. El motivo de lo anterior fue para tener dos periodos de 117 semanas cada uno y comparar cual era el comportamiento de la beta desapalancada antes y después de diciembre de 2011 , fecha en que las empresas se incorporarán al índice sustentable del IPC, para evaluar si hubo cambio o no el riego de negocio para cada una de las empresas antes versus posterior a su incorporación al IPC sustentable.

Para el cálculo de los coeficientes beta se determinaron por el método de regresión lineal, en donde la variable independiente $X$ es el rendimiento semanal de mercado $\left(R_{m}\right)$ y la variable dependiente $Y$ es el rendimiento semanal de la acción $\left(R_{J}\right)$ de la empresa correspondiente, con la fórmula:

$$
R_{J}=\propto+\beta R_{m}+\varepsilon_{j}
$$


Dónde $\alpha=$ intercepto de la regresión, y $\beta=$ pendiente de la ecuación de regresión, y el valor de la pendiente es considerado el coeficiente beta de la acción y cuyo cálculo también puede ser determinado por la covarianza:

$$
B_{t}=\frac{\operatorname{Cov}\left(R_{j}, R_{m}\right)}{\operatorname{Var}\left(R_{m}\right)}
$$

Para estimar la beta desapalancada $B_{U}$, se utilizó la fórmula desarrollada por Hamada (1969) y aplicada por Damodaran (1998) y otros más como: Vélez (2011) y Moscoso et. al., (2012), Hernández y Mora (2010) en diferentes estudios, y la fórmula es:

$$
B_{U}=\frac{B}{\left[1+\frac{(1-t) \text { Deuda Financiera }}{\text { Patrimonio }}\right]}
$$

Dónde

\begin{tabular}{|l|l|l|}
\hline $\mathrm{B}_{\cup}$ & $=$ & Beta de los activos o beta desapalancada \\
\hline $\mathrm{B}$ & $=$ & Beta de la empresa obtenida por el método de mínimos cuadrados \\
\hline $\mathrm{t}$ & $=$ & Tasa fiscal \\
\hline
\end{tabular}

Dónde $B$ es la beta del precio de la acción de la empresa por regresión, t es la tasa marginal de impuestos y la deuda financiera son todos los pasivos por los cuales se pagan un costo explícito.

\subsubsection{Método estadístico}

Para conocer el contraste entre los valores promedios de las betas desapalancadas se calculó la diferencia entre los valores medios de las betas desapalancadas de dos muestras, la primer muestra pertenece al periodo 2009-2011 y es el periodo previo al ingreso al índice sustentable, y se comparó con la segunda muestra que fue el periodo de 2012-2014, es decir, después de que las empresas se certificaron para ingresar al índice sustentable, estos cálculos se hicieron para cada una de las empresas que integran el índice sustentable. Primero se calcula la diferencia entre cada par de valores y se determina la media $\bar{D}$ y la desviación estándar $S_{D}$ de las diferencias de medias muestrales, posteriormente se calcula el intervalo de confianza del $95 \%$ de dos colas para la diferencia del valor medio, con las fórmulas:

$$
\bar{D}=\frac{\sum D_{i}}{n} \quad S_{D}=\sqrt{\frac{\sum D_{i}^{2}-n \bar{D}^{2}}{n-1}} \quad \bar{D} \pm t \propto / 2 \frac{S_{D}}{\sqrt{N}}
$$


Dónde:

\begin{tabular}{|l|l|l|}
\hline $\mathrm{S}_{\mathrm{D}}$ & $=$ & Desviación estándar de la diferencia de medias de dos grupos \\
\hline $\mathrm{D}^{2}$ & $=$ & Cuadrado de la diferencia apareada \\
\hline $\mathrm{n}$ & $=$ & Número de pares muestrales apareados \\
\hline $\bar{D}$ & $=$ & Media de las diferencias apareadas \\
\hline$\mu$ & $=$ & Media del grupo “i” \\
\hline
\end{tabular}

Debido a que el error estándar de la diferencia media se calcula con base en la desviación estándar de la muestra de diferencias, y ya que en general se puede suponer que los valores $D$ tienen una distribución normal, la distribución apropiada para probar la hipótesis nula es la distribución $t$.

El estadístico de prueba para validación de hipótesis con muestras apareadas sigue la distribución t, y su valor se calcula a partir de la siguiente fórmula:

$$
t=\frac{\bar{D}}{S_{D} \div \sqrt{n}}
$$

Dónde $\bar{D}$ es la media de la diferencia entre las observaciones apareadas, $\mathrm{S}_{\mathrm{D}}$ el error estándar de la media, $\mathrm{n}$ el número de observaciones.

Las pruebas de dos muestras se utilizan para evaluar si las medias de dos poblaciones son iguales o diferentes, para ello se requieren dos muestras independientes, una por cada una de las dos poblaciones, en este caso la primer población se integró con los valores semanales de las betas desapalancadas por cada una de las empresas antes de ingresar al índice sustentable de la BMV y la segunda fue el grupo de valores de betas semanales de cada una de las empresas posteriores al ingreso al IPC sustentable, lo cual permitió evaluar si hubo cambio en el riesgo de negocio de cada una de las empresas del índice sustentable de la BMV.

\section{Resultados y discusión}

En la Tabla 5 se observan los resultados del valor de la beta desapalancada del periodo previo (2009-2011), versus el periodo posterior (2012-2014) a la incorporación al índice sustentable de la BMV. En el periodo 2009-2011, solo tres empresas tienen una beta mayor de 1, CEMEX, GMÉXICO y PEÑOLES, ocurre lo mismo para el periodo que comprende de 2012-2014, es decir, que estas empresas son las que tienen el mayor riesgo del total de las compañías estudiadas, cabe resaltar que pertenecen al sector Materiales. 
Cuando se compara el valor promedio de las betas de los dos periodos se observa que AMX, ASUR, BIMBO, CEMEX, FEMSA, GMODELO, HOMEX, ICA, KOF, OMA, PEÑOLES y URBI disminuyen en su coeficiente de beta desapalancada, es decir, su riesgo de negocio, algunas de estas empresas se encuentran en mercados que son considerados monopolios u oligopolios, estas compañías son preponderantes,

Por otra parte en la validación de la hipótesis se encontró que en 17 compañías se rechaza la hipótesis nula, es decir, que si existe diferencia significativa en el valor medio de la beta desapalancada entre el periodo previo versus a la incorporación al índice sustentable de la BMV, de estas empresas solo 12 compañías disminuyeron en su coeficiente de riesgo de negocio, medido a través de la beta desapalancada.

\section{Conclusiones y Recomendaciones}

En este trabajo se calculó el riesgo de negocio, mediante el coeficiente de las betas desapalancadas de 20 empresas que integran el índice sustentable de la Bolsa Mexicana de Valores que comenzó a operar en el año 2011, se compararon dos periodos, el 2009-2011 versus 2012-2014, para medir los cambios en el riesgo de negocio de las empresas como resultado de haberse certificado como sustentables. Se encontró que del total de las 20 empresas, 12 empresas disminuyeron el riesgo de negocio, lo cual resulta incongruente con los postulados de las empresas sustentables, porque se considera que por adoptar las certificaciones que exigen las bolsas de valores para incluirse en el índice bursátil sustentable, deberían caracterizarse por ser empresas más robustas para enfrentar los embates del medio ambiente.

De las 8 empresas que incrementaron su nivel de riesgo operativo, pertenecen a diferentes sectores, como es el sector de consumo frecuente, controladoras y materiales, esto quiere decir que la participación de cada una de estas empresas en el mercado es determinante en los flujos de efectivo que obtienen y por consecuencia su nivel de riesgo, pues otras empresas que pertenecen a estos mismos sectores si disminuyeron su nivel de riesgo, donde seguramente son preponderantes como es el caso de GMODELO, del sector de consumo frecuente. Por otra parte algunas compañías han tenido problemas en el pasado que afecta aún los resultados de sus operaciones, es el caso de COMERCI, que durante el año 2009, tuvo problemas por el manejo de instrumentos financieros derivados, en el caso de GEO que pertenece a la rama de industrial específicamente la construcción de vivienda en el año 2014 se han presentado problemas en el mercado de viviendas, de tal suerte que el efecto de la tendencia del sector ha influido en el aumento de su riesgo operativo. 
Enfoque UTE, V.5-N.4, Dic.2014: pp.17 - 33

Copyright (C) 2014 Universidad Tecnológica Equinoccial

http://ingenieria.ute.edu.ec/enfoqueute/

ISSN: 1390-6542
Recibido (Received): 2014/10/27

Aceptado (Accepted): 2014/12/15

Tabla 5. Prueba de hipótesis

Beta (09.10.2009 al 31.12.2011 versus 06.01.2012 al 28.03.2014)

\begin{tabular}{|c|c|c|c|c|c|c|c|c|c|c|}
\hline \multirow{2}{*}{ No. } & \multirow{2}{*}{ Acción } & \multicolumn{2}{|c|}{ Promedio $\mathrm{Bu}=$ beta } & \multirow{2}{*}{$\begin{array}{c}\text { Media de la } \\
\text { diferencia apareada }\end{array}$} & \multirow{2}{*}{\multicolumn{2}{|c|}{ Intervalo de confianza }} & \multirow{2}{*}{$\begin{array}{l}\text { Limite } \\
\text { inferior }\end{array}$} & \multirow{2}{*}{$\begin{array}{l}\text { Limite } \\
\text { superior }\end{array}$} & \multirow{2}{*}{$\begin{array}{c}\text { Valor calculado de la } \\
\text { estadistica "t" de prueba }\end{array}$} & \multirow{2}{*}{ Validación de la hipótesis } \\
\hline & & $2009-2011$ & $2012-2014$ & & & & & & & \\
\hline 1 & AC & 0.3200 & 0.6199 & -0.2999 & \pm & 0.0281 & -0.3281 & -0.2718 & -20.8855 & Rechaza \\
\hline 2 & ALFA & 1.0556 & 0.9958 & 0.0597 & \pm & 0.0639 & -0.0041 & 0.1236 & 1.8334 & Acepta \\
\hline 3 & AMX A & 0.7508 & 0.8404 & -0.0896 & \pm & 0.0341 & -0.1238 & -0.0555 & -5.1441 & Rechaza \\
\hline 4 & ASUR B & 0.6112 & 0.5922 & 0.0190 & \pm & 0.0430 & -0.0241 & 0.0620 & 0.8631 & Acepta \\
\hline 5 & BIMBO A & 0.8540 & 0.8224 & 0.0316 & \pm & 0.0378 & -0.0062 & 0.0694 & 1.6388 & Acepta \\
\hline 6 & CEMEX CPO & 1.9463 & 2.1416 & -0.1953 & \pm & 0.1220 & -0.3174 & -0.0733 & -3.1371 & Rechaza \\
\hline 7 & COMERCI UBC & 1.1035 & 0.7848 & 0.3187 & \pm & 0.0765 & 0.2422 & 0.3951 & 8.1648 & Acepta \\
\hline 8 & :FEMSA & 0.1421 & 0.2804 & -0.1383 & \pm & 0.0363 & -0.1747 & -0.1020 & -7.4576 & Rechaza \\
\hline 9 & GEO B & 1.1418 & 1.3723 & 0.1566 & \pm & 0.1114 & 0.0452 & 0.2680 & 2.7558 & Rechaza \\
\hline 10 & GMEXICO & 1.2975 & 1.3426 & -0.0450 & \pm & 0.0676 & -0.1126 & 0.0225 & -1.3061 & Acepta \\
\hline 11 & GMODELO & 0.4392 & 0.3914 & 0.0478 & \pm & 0.0334 & 0.0144 & 0.0812 & 2.8019 & Rechaza \\
\hline 12 & HOMEX & 1.5030 & 1.2846 & 0.2513 & \pm & 0.0738 & 0.1775 & 0.3252 & 6.6711 & Rechaza \\
\hline 14 & KIMBER & 0.6441 & 0.7662 & -0.1221 & \pm & 0.0353 & -0.1574 & -0.0867 & -6.7685 & Rechaza \\
\hline 15 & KOF & 0.4924 & 0.4613 & 0.0311 & \pm & 0.0543 & -0.0232 & 0.0854 & 1.1227 & Acepta \\
\hline 16 & MEXCHEM & 0.1969 & 0.1969 & 0.0000 & \pm & 0.0000 & 0.0000 & 0.0000 & 0.0000 & Rechaza \\
\hline 17 & OMA & 0.8507 & 0.3243 & 0.5264 & \pm & 0.0676 & 0.4588 & 0.5940 & 15.2665 & Rechaza \\
\hline 18 & :PEÑOLES & 1.4701 & 1.1808 & 0.2893 & \pm & 0.0618 & 0.2275 & 0.3512 & 9.1727 & Rechaza \\
\hline 19 & URBI & 1.3274 & 0.9605 & 0.6460 & \pm & 0.0658 & 0.5803 & 0.7118 & 19.2484 & Rechaza \\
\hline 20 & $\begin{array}{l}\text { WALMEX } \\
\end{array}$ & 0.7154 & 0.7622 & -0.0468 & \pm & 0.0293 & -0.0760 & -0.0175 & -3.1343 & Rechaza \\
\hline
\end{tabular}

Para una prueba de dos colas basada en $n=$ infinito observaciones, y $a=05$, se asigna 0.025 a cada cola de distribución de $t$ y No se aceptará cuando $t>1.96$ ó $t<-1.96$.

Fuente: Elaboración propia con resultados de la investigación. 
Por otra parte, las empresas estudiadas en este trabajo apenas tienen dos años y medio de haberse certificado como sustentables, por lo cual probablemente aún no logran consolidarse en sus mercados mediante la reputación que se asocia a las empresas sustentables, por lo que es recomendable que se vuelvan a evaluar posteriormente en su riesgo específico, por lo cual no se llega a una conclusión definitiva, sino que es necesario más estudios, que sólo en los años venideros se podrá tener mejor información para determinar en el caso de México si se cumplen o no las hipótesis.

\section{Agradecimientos}

A la Escuela Superior de Comercio y Administración unidad Tepepan del Instituto Politécnico Nacional, al Sistema Nacional de Investigadores del Consejo Nacional de Ciencia y Tecnología y a la revista ENFOQUE.

\section{Bibliografía}

Almagro F. (2009). Cuentas ecológicas y desarrollo sustentable. La experiencia en México, México, Instituto Politécnico Nacional.

Berk J. y Demarzo P. (2008). Finanzas corporativas, México: Pearson.

Brealey R. A. y Myers S. A. (1998). Principios de finanzas corporativas, España: McGraw-Hill.

Centro Empresarial de Inversión Social (2004). La Responsabilidad Social Empresarial. Una prioridad en el Mundo Empresarial Moderno, disponible en: http://www. cepal.org/celade/noticias/paginas/8/12168/respempresarialglobal.pdf [Consultado: Noviembre 2013]

Damodaran Aswath, (1998), "Models of Risk" en Berstein, Peter y Damodaran, Aswath: Investment Management, Jhon Wiley, Nueva York, pp. 58-80.

Deloitte, (2011), Sustentabilidad como herramienta para reducir los riesgos, Boletín Gobierno Corporativo, Deloitte.

Deloitte, (2011). Sustentabilidad como herramienta para reducir riesgos. Boletín Gobierno Corporativo, verano, disponible en: http://www2.deloitte.com/content/dam/Deloitte/mx/ Documents/risk/Gobierno-Corporativo/gc-sustentabilidad-herramienta-reducir-riesgos-vera no2011.pdf

Espino G. M. (2009). Sustentabilidad de las empresas, México, Instituto Mexicano de Contadores Públicos. 
Forética, (2011), Sostenibilidad par a financieros, RSEARCH Publicación de tendencias y actualidad RSE, Junio, No. 4. Madrid, pp. 31., disponible en: http://www.spainsif.es/ sites/default/files/upload/varios/sostenibilidad_para_financieros.pdf [Consultado: noviembre 2013]

Gitman L. J. (2007). Principios de Administración financiera, México: Pearson.

Gitman L. J. y Joehnk M. D. (1997). Fundamentos de inversión, México: Oxford University PressHARLA.

Hamada, R. S. (1969). Portfolio Analysis, Market Equilibrium and Corporation Finance. The Journal of Finance, Vol. 24, No. 1, 13-31.

Hernández R. M. y Mora E. R. (2009). Propuesta de un modelo dinámico de asignación de precios de activos para valorar proyectos y empresas en mercados emergentes: El caso de Costa Rica, InerSedes: Revista de las Sedes Regionales, vol. X, núm. 19, Universidad Costa Rica, pp. 72-88.

Hernández R. M. y Mora E. R. (2010). Diseño de un modelo de estimación de retornos ajustados por riesgo para actividades de valoración de mercados emergentes, TEC Empresarial, Volumen 4, No. 1, abril, Instituto Tecnológico de Costa Rica, pp. 49-56

Koontz H., Weihrich H. y Cannice M., (2012), Administración. Una perspectiva global empresarial, México, McGraw-Hill.

Lacruz M. F. (2005). La empresa ambientalmente responsable. Una visión de futuro, Economía, número 2, enero-diciembre, pp. 39-58.

Lintner, J. (1965). The valuation of risky assets and the selection of risky investments in stock portfolio and capital budgets. Review of Economics and Statistics, Vol. 47, N 1, 13-37.

Little J. B. y Rhodes L. (1992). Cómo entender a Wall Street, México: McGraw-Hill.

López H. F. (2000). ¿Es igual el riesgo sistémico al medir la beta CAPM mediante dos indicadores diferentes del mercado? Estudio empírico en la Bolsa Mexicana de Valores, Contaduría y Administración, No. 197, abril-junio, pp. 5-15.

Malkiel B. G. (1995), Un paseo aleatorio por Wall Street, Madrid: Alianza Editorial.

Margolis J. D. y Walsh J. P., (2001), Misery Loves Companies: Whither Social Initiatives by Business. Harvard Business School, June, pp. 1-62.

Moscoso E. J., Sepúlveda R. C., García C. A. y Restrepo L. A. (2012). Costo de capital en entornos económicos cambiantes: Caso Valle de Aburrá (Antioquía), en Revista Facultad de 
Ciencias Económicas Investigación y Reflexión, Vol. XX, No. 2, Bogotá, julio-diciembre, pp. 189-202.

Mossin, J. (1966). Equilibrium in a capital asset market. Econometrica, Vol. 34, № 4, 768-783.

Murillo L. J., Garcés A. C. y Rivera T. P. (2004), "Estrategia empresarial y medio ambiente: opinión de un grupo de expertos", Universia Bussiness Review -Actualidad Económica, No. 4, disponible en: http://www.redalyc.org/pdf/433/43300405.pdf. [Consultado: junio 2014].

Nieto Antolín Mariano y Fernández Gago Roberto. (2004), Responsabilidad social corporativa: última innovación en management. Universia Bussiness Review- Actualidad Económica, primer trimestre, número 1, pp. 28-39.

Núñez G. (2003). La responsabilidad social corporativa en un marco de desarrollo sostenible, Santiago de Chile, Comisión Económica para América Latina de las Naciones Unidas.

Organización Internacional del Trabajo (OIT) (2007). La promoción de las empresas sostenibles, Suiza, Oficina Internacional de Trabajo.

Orlitzky M., Schmidt F. y Rynes S. (2003), Corporate Social and Financial Performance: A Metaanalysis, Organization Studies, 24(3): 403-441.

Páez H. (2013). Teoría económica sobre evaluación ambiental de proyectos, México, Instituto Politécnico Nacional.

Perera L. (2011), Las instituciones del mercado financiero. En Banco Interamericano de Desarrollo, (coord), La responsabilidad Social de la empresa en América Latina, (pp. 161193), Washington D.C., Banco Interamericano de Desarrollo.

Ponce P. y Pérez F. (2007). La economía y el medio ambiente, en Figueroa H., Tavera M. y Pérez F., (coordinadores), Desarrollo sustentable y calidad de vida, México, Universidad Autónoma de Chapingo.

Porter M. y Kramer M. (2006), Estrategia y Sociedad. Harvard Business School America Latina, diciembre, pp. 1-15.

Porter M. y Kramer M. (2011), Creating Shared value. Harvard Business School, JanuaryFebruary, pp. 1-17.

Senior A., Narváez M., Fernández G. y Revilla J. (2007), Responsabilidad ambiental: factor creador de valor agregado en las organizaciones, Revista en Ciencias Sociales Vol. XIII, No. 3 Septiembre-diciembre, pp. 484-494. 
Sharpe, W. F. (1964). Capital asset prices: a theory of market equilibrium under conditions of risk. Journal of Finance, Vol. 19, № 2, 425-442.

Stoner J., Freeman R. y Gilbert D. (1996). Administración, México, Pearson Prentice Hall.

Valderrábano M., Castro J., Trujillo M. y Hernández R. (2011), Cuaderno de política ambiental, México, Instituto Politécnico Nacional.

Van Horne J. C. (1997). Administración financiera, México: Prentice Hall.

Vargas S. A. (2011). Estimación del costo del patrimonio y costo del capital por medio de tasas ajustadas al riesgo, Investigación y Desarrollo, No. 11, marzo, Universidad Privada Boliviana, pp. 118-135.

Vélez P. I. (2011). Estimación de betas y relación entre las betas apalancadas y el coste de capital, Análisis financiero, No. 116, segundo cuatrimestre, Instituto Español de Analistas Financieros, pp. 6-13.

Weston J. F. y Brigham E. F. (1993). Fundamentos de administración financiera, México: McGrawHill.

\section{Páginas web y fuentes de datos especializados}

\section{Economática}

Buenaventura, (2014), Departamento de Estudios Contables y Financieros, Facultad de Ciencias Administrativas y Económicas de La Universidad ICESI de Cali Colombia en: http://www.icesi.edu.co/departamentos/finanzas_contabilidad/betas_colombia.php 